\title{
Semaglutide Injection For the Treatment of Adults with Type 2 Diabetes
}

RA Chudleigh(1) \& SC Bain(1,2)

Singleton Hospital, Swansea Bay University Health Board(1) and Diabetes Research Unit, Swansea University Medical School, UK(2)

\section{Structured abstract}

This article examines the use of injectable semaglutide in the treatment of type 2 diabetes. A brief description of the global problem posed by type 2 diabetes is followed by consideration of the glucagon-like peptide 1 receptor agonist class of glucose lowering therapies. The focus is then shifted to semagltuide and a description of the large phase 3 pre-approval trial programme known as SUSTAIN. There is consideration of glucose control, the primary end-point of the phase 3 programme as well as secondary end-points such as weight and blood pressure. There follows a précis of the cardiovascular outcomes trial for subcutaneous semaglutide (SUSTAIN 6) and the post-approval publications. The expert opinion section discusses the position of semaglutide and the pros and cons versus other once weekly GLP-1RAs and finally the development of an oral version of semaglutide, which has recently been approved in the United States.

\section{Introduction}

Diabetes mellitus (DM) is a common group of metabolic diseases, which are characterised by persistent elevation of plasma glucose levels. The two major forms of DM are termed type 1 and type 2 and both are associated with development of microvascular complications affecting the eyes, kidneys and nerves (termed retinopathy, nephropathy and neuropathy) as well as an increased risk of cardiovascular (CV) disease. Type 2 DM (T2DM) is characterized by insulin resistance and progressive loss of insulin secretion by beta-cells in the pancreatic islets of Langerhans. (REF 1). The underlying driver for T2DM in most cases is being overweight or obese and the increasing prevalence of obesity is the reason that the incidence continues to rise. Termed the 'diabetes epidemic', it is estimated that around $10 \%$ of the global adult population are affected by T2DM (REF 2). In theory, lifestyle modification should both prevent and be an effective treatment for T2DM but in real life changes in diet and exercise are difficult to implement. This has promoted the development of pharmacologic glucose lowering therapies and in Europe there are currently eight different classes of these medications.

The glucagon-like peptide 1 (GLP-1) receptor agonist class of glucose lowering medicines was introduced into clinical practice in 2006. These agents stimulate the GLP-1 receptor and this enhances pancreatic insulin secretion whilst reducing the production of glucagon, both in a glucose-dependent manner. GLP-1 receptor agonists (GLP-1RAs) can also slow gastric emptying and, probably via an effect on the central nervous system, increase satiety, both of which can promote weight loss. These agents are currently available as injectable peptides which, unlike endogenous GLP-1, are resistant to degradation by the ubiquitous enzyme dipeptidyl peptase-4 (DPP-4). The major side-effects of this class are gastro- 
intestinal (GI) with nausea, vomiting and diarrhoea being most common (although these typically diminish over time).

Exenatide was the first GLP-1RA to be launched (Byetta ${ }^{\circledR}$ ) and was based on the exendin-4 molecule; due to its short half-life it was administered twice daily with meals (REF 3 ). In 2011 the European Medicines Agency authorised a once-weekly (QW) version of exenatide [Bydureon ${ }^{\circledR}$ ], the first long-acting GLP-1RA (REF 4) and in 2020 there are two other commercially available once weekly GLP-1RA therapies, dulaglutide (REF 5) and semaglutide (REF 6).

Semaglutide (Ozempic ${ }^{\circledR}$ ) was approved by the United States Food and Drug Administration (FDA) in 2017 and by the European Medicines Agency (EMA) in 2018, as an addition to diet and exercise in people with poorly controlled T2DM (REF 7). Unlike exenatide, this molecule is based on human GLP-1 and has $94 \%$ homology with this molecule, differing only by two amino acids. The substitution at position 8 (alanine to alpha-aminoisobutyric acid) protects against DPP4 degradation whilst an 18 carbon fatty di-acid chain attached via a spacer to the lysine amino acid at position 26 provides strong binding to albumin (figure 1). These modifications facilitate the prolongation of action, with an elimination half-life of approximately 168 hours. Semaglutide is delivered as a clear, colourless solution which does not require resuspension. It is initiated at a dose of $0.25 \mathrm{mg} \mathrm{QW}$ titrated after four weeks to a maintenance dose of $0.5 \mathrm{mg}$ or, with further up-titration, $1.0 \mathrm{mg} \mathrm{QW}$. Semaglutide is extensively metabolized and the metabolites are predominantly excreted by the renal tract and to a lesser extent in the faeces (REF 8) Exposure to semaglutide is not significantly affected by severe renal impairment, nor impaired liver function (REF 9).

\section{Pharmacodynamics, pharmacokinetics and metabolism of semaglutide}

\section{Absorption}

In healthy adults, subcutaneous administration of one dose of semaglutide $0.5 \mathrm{mg}$ reaches a maximum concentration ( $\mathrm{Cmax}$ ) in 24-56 hours (REFS 8,9). Dose-escalation studies using weekly semaglutide $0.25 \mathrm{mg}$ doses for four weeks, followed by $0.5 \mathrm{mg}$ (four weeks) and then $1.0 \mathrm{mg}$ (five weeks) showed a similar time to Cmax of 33-36 hours after the final 1.0 mg dosing (REFS 10,11). Cmax and area under the plasma concentration-time curve (AUC) are similar following a single $0.5 \mathrm{mg}$ dose and following the final $1.0 \mathrm{mg}$ dose during a dose escalation (REFS $8,9,10,11$ ). Subcutaneous bioavailability of semaglutide is $94 \%$, which is the highest of all currently available GLP-1 RAs (REFS 12,13,14,15).

\section{Distribution}

Semaglutide was intended to be an analogue of liraglutide with increased binding affinity for albumin, so as to allow for once-weekly dosing. Lau et al. showed in living pigs that the half-life of semaglutide following intravenous administration was approximately 46 hours compared with 12 hours for liraglutide (REF 12). Semaglutide had a higher volume of distribution $(0.102 \mathrm{~L} / \mathrm{kg}$ versus $0.067 \mathrm{~L} / \mathrm{kg})$ and slower clearance $(0.0016 \mathrm{vs} .0 .0038 \mathrm{~L} / \mathrm{h} / \mathrm{kg})$, indicative of enhanced binding to albumin. Mean residence time following subcutaneous 
administration of semaglutide was around 63 hours compared with 23 hours for liraglutide but there were no toxic effects resulting from the longer exposure to drug (REF 12).

Metabolism

The metabolism of semaglutide was studied in an absorption, metabolism, and excretion study in seven healthy males who received a single subcutaneous dose of radiolabeled [3H]semaglutide (REF 8). Metabolite profiling showed that semaglutide is metabolized to six different metabolites labelled P1-P3 and P5-P7. P4 was the parent compound [3H]semaglutide and this was the primary component detected in plasma (82.6\%). Semaglutide is metabolized by proteolytic cleavage of the peptide backbone and beta-oxidation of its fatty acid side chain. The concentrations of the metabolites declined over time, with only the parent compound being detected in plasma at 28 days after dosing. It is unknown if the metabolites play any role in the efficacy or adverse effects of semaglutide.

\section{Elimination}

In same study, the excretion of radiolabeled [3H]-semaglutide and its metabolites was also reported (REF 8). After 64 days of collection following the single subcutaneous dosing of $0.5 \mathrm{mg}$ semaglutide, $75 \%$ of the dose was recovered. $53 \%$ was in urine, $18.6 \%$ in faeces and $3.2 \%$ in expired air. The parent semaglutide molecule and 21 metabolites were detected in urine, and around $3 \%$ of the administered dose was found to be $[3 \mathrm{H}]$-semaglutide, with two metabolites (P6 and P7) each accounting for 14\% of the administered dose, and all other metabolites accounting for $<2 \%$ of the administered dose. The minimal amount of intact drug in the urine indicated that adjustment of dosing according to renal status would not be necessary. No parent drug was detected in faeces.

In a phase 2 clinical trial, four hundred and fifteen subjects were randomised to subcutaneous semaglutide without dose escalation (0.1-0.8 $\mathrm{mg} \mathrm{QW}$ ) for twelve weeks or with dose escalation ( $0.4 \mathrm{mg}$ steps to 0.8 or $1.6 \mathrm{mg}$ over $1-2$ weeks) (REF 16). The primary end-point was change in $\mathrm{HbA1c}$ from baseline; secondary end-points included change in body weight, safety, and tolerability. Semaglutide dose-dependently reduced HbA1c from baseline $(65 \mathrm{mmol} / \mathrm{mol}$ ) by up to $18.6 \mathrm{mmol} / \mathrm{mol}$ and reduced body weight by as much as $4.8 \mathrm{~kg}$. Over $80 \%$ of patients achieved an $\mathrm{HbA} 1 \mathrm{c}<53 \mathrm{mmol} / \mathrm{mol}$. An open-label comparison with liraglutide $1.2 \mathrm{mg}$ and $1.8 \mathrm{mg} \mathrm{OD}$ in the same study showed that $\mathrm{HbA} 1 \mathrm{c}$ and weight reduction with semaglutide $1.6 \mathrm{mg}$ were better but adverse events (AEs) and withdrawals were more frequent (REF 16). The incidence of nausea, vomiting, and withdrawal due to gastrointestinal AEs increased with semaglutide dose although most events were mild-tomoderate, transient, and ameliorated by dose escalation. There were no major episodes of hypoglycaemia, few injection site reactions and no unexpected safety or tolerability concerns. On this basis, weekly semaglutide doses of 0.5 and $1.0 \mathrm{mg}$ with a 4-week dose escalation were selected for the phase 3 assessments.

\section{The phase 3 clinical trial programme for semaglutide (table)}

\section{Glucose control (figure 2)}


Phase 3 clinical trials of semaglutide were termed the 'Semaglutide Unabated Sustainability in Treatment of Type 2 diabetes' (SUSTAIN) programme and included 8,416 people with T2DM (REF 17). SUSTAIN was made up of six trials, each with a primary endpoint of change in glucose control (assessed by HbA1c) from baseline to the end of trial (EOT). In addition, a cardiovascular outcome trial (CVOT) was performed. Semaglutide was assessed in patients across the entire spectrum of T2DM, from treatment-naïve, through combinations with oral glucose lowering agents (metformin, sulphonylureas and thiazolidinediones) and with insulin. All of the studies were randomised controlled trials (RCTs) assessing the glucose lowering efficacy of semaglutide compared to placebo, DPP-4 inhibitor, GLP-1RAs and longacting insulin.

\section{SUSTAIN 1}

In this study, semaglutide was compared with placebo injection in people with T2DM treated with only diet and exercise (i.e. not receiving any other glucose lowering therapies)(REF 18). Participants had an average diabetes duration of 4.2 years and mean body mass index (BMI) of $32.9 \mathrm{~kg} / \mathrm{m}^{2}$. The mean baseline $\mathrm{HbA1c}$ was $64.5 \mathrm{mmol} / \mathrm{mol}$, which after 30 weeks fell by $16.0 \mathrm{mmol} / \mathrm{mol}$ with semaglutide $0.5 \mathrm{mg}$ QW and by $17.1 \mathrm{mmol} / \mathrm{mol}$ with semaglutide $1.0 \mathrm{mg} \mathrm{QW}$, both reductions being significantly greater than placebo $(-0.2$ $\mathrm{mmol} / \mathrm{mol}$ ). This led to significantly more subjects achieving an $\mathrm{HbA} 1 \mathrm{c}<53 \mathrm{mmol} / \mathrm{mol}$ with semaglutide $0.5 \mathrm{mg}$ and $1.0 \mathrm{mg}$ ( $74 \%$ and $72 \%$ respectively) compared with placebo (25\%).

\section{SUSTAIN 2}

In the SUSTAIN 2 study, semaglutide $0.5 \mathrm{mg}$ and $1.0 \mathrm{mg}$ QW were compared with oral sitagliptin $100 \mathrm{mg}$ (a DPP4-inhibitor) once daily (OD) over a 56 week period (REF 19). Subjects were being treated with other glucose lowering therapies (predominantly metformin) and had a baseline $\mathrm{HbA1c}$ of $64.1-65.8 \mathrm{mmol} / \mathrm{mol}$. The average duration of T2DM was $6.4-6.7$ years and mean BMI was $32.5 \mathrm{Kg} / \mathrm{m}^{2}$. At EOT the HbA1c was significantly lower in the semaglutide $0.5 \mathrm{mg}(14.4 \mathrm{mmol} / \mathrm{mol})$ and $1.0 \mathrm{mg}(17.6 \mathrm{mmol} / \mathrm{mol})$ groups compared with a $6.0 \mathrm{mmol} / \mathrm{mol}$ reduction with sitagliptin.

\section{SUSTAIN 3}

This trial was a comparison of semaglutide $1.0 \mathrm{mg}$ QW with the once-weekly GLP-1RA exenatide extended release (exenatide ER) $2.0 \mathrm{mg}$ (REF 20). Participants were also treated with other glucose lowering therapies, mainly metformin (96.3-96.8\%) and sulphonylureas (44.8-51.4\%). The average diabetes duration of trial subjects was 9.2 years and mean BMI was $33.8 \mathrm{~kg} / \mathrm{m}^{2}$. At EOT (56 weeks) $\mathrm{HbA} 1 \mathrm{c}$ was reduced by $16.8 \mathrm{mmol} / \mathrm{mol}$ in the semaglutide arm compared with $10.0 \mathrm{mmol} / \mathrm{mol}$ for exenatide ER group and this difference was statistically significant in favour of semaglutide.

\section{SUSTAIN 4}

Here, semaglutide was compared with the long-acting insulin analogue, insulin glargine (U100 concentration (REF 21). People with T2DM who were sub-optimally controlled on a stable dose of metformin and/or sulphonylureas (but naïve to insulin) were assigned to semaglutide $0.5 \mathrm{mg}$ or $1.0 \mathrm{mg}$ QW or glargine. The starting dose of glargine was 10 units OD and this was titrated to a fasting plasma glucose target of $4.0-5.5 \mathrm{mmol} / \mathrm{l}$. Trial recruits had an average duration of diabetes of 8.6 years and a mean $\mathrm{BMI}$ of $33.0 \mathrm{Kg} / \mathrm{m}^{2}$. The mean $\mathrm{HbA} 1 \mathrm{c}$ at baseline was $65.8 \mathrm{mmol} / \mathrm{mmol}$ and at EOT (30 weeks) fell by $13.2 \mathrm{mmol} / \mathrm{mol}$ with 
semaglutide $0.5 \mathrm{mg} \mathrm{QW}$ and $17.9 \mathrm{mmol} / \mathrm{mol}$ with semaglutide $1.0 \mathrm{mg} \mathrm{QW}$. Both of these reductions were significantly better than that achieved by glargine $(9.1 \mathrm{mmol} / \mathrm{mol})$ although the mean insulin dose was modest ( 29.2 units OD) and the achieved fasting plasma glucose was $7.1 \mathrm{mmol} / \mathrm{l}$ (i.e. above the pre-specified target).

\section{SUSTAIN 5}

In the SUSTAIN 5 study, semaglutide $0.5 \mathrm{mg} \mathrm{QW}$ and $1.0 \mathrm{mg}$ QW were investigated as an add-on to basal insulin therapy and compared with placebo (REF 22). Trial recruits were already treated with a stable dose of basal insulin (neutral protamine Hagedorn [NPH], glargine, detemir or degludec) either alone or in combination with metformin. The average diabetes duration was 13.3 years and daily basal insulin doses were 36.6-39.3 units. In those subjects with baseline $\mathrm{HbA1c}$ less than $64 \mathrm{mmol} / \mathrm{mol}$, the insulin dose was reduced by $20 \%$ so as to avoid hypoglycaemia; the insulin dose could then be up-titrated between weeks 10 and 16. The mean baseline $\mathrm{HbA1c}$ was $67.9 \mathrm{mmol} / \mathrm{mol}$ and at EOT (30 weeks) fell significantly more with semaglutide $0.5 \mathrm{mg} \mathrm{QW}(15.8 \mathrm{mmol} / \mathrm{mol})$ and $1.0 \mathrm{mg} \mathrm{QW}(20.2$ $\mathrm{mmol} / \mathrm{mol})$ than with placebo $(1.0 \mathrm{mmol} / \mathrm{mol})$. These improvements in $\mathrm{HbA} 1 \mathrm{c}$ were seen despite their being a reduction in insulin dose from baseline in both the semaglutide groups (0.5mg QW; mean 39.3 to 35.4 units OD and $1.0 \mathrm{mg} \mathrm{QW} ; 37.4$ to 31.5 units OD).

\section{SUSTAIN 7}

The SUSTAIN 7 trial was another head-to-head comparison with a weekly GLP-1RA, this time dulaglutide(REF 23). People with T2DM with suboptimal glycaemic control on metformin (minimum dose of $1.5 \mathrm{~g} \mathrm{OD}$ ) were randomised to semaglutide $0.5 \mathrm{mg}$ or $1.0 \mathrm{mg} \mathrm{QW}$, or dulaglutide $0.75 \mathrm{mg}$ or $1.5 \mathrm{mg} \mathrm{QW}$. The average diabetes duration was 7.0-7.7 years and mean BMI was $33.1-33.7 \mathrm{~kg} / \mathrm{m}^{2}$. The baseline mean $\mathrm{HbA} 1 \mathrm{c}$ was $66.1-67.2 \mathrm{mmol} / \mathrm{mol}$ and at EOT (40 weeks) was significantly reduced in all four treatment cohorts. Comparison of the low and high doses of each agent showed a significant benefit in glucose lowering for semaglutide over dulaglutide (semaglutide $0.5 \mathrm{mg} \mathrm{QW} 16.5 \mathrm{mmol} / \mathrm{mol}$ versus dulaglutide $0.75 \mathrm{mg} \mathrm{QW} 12.1 \mathrm{~mol} / \mathrm{moland}$ semaglutide $1.0 \mathrm{mg} \mathrm{QW} 19.4 \mathrm{mmol} / \mathrm{mol}$ versus dulaglutide 1.5 $\mathrm{mg} 14.9 \mathrm{mmol} / \mathrm{mol})$.

\section{Secondary endpoints - Body weight (figure 3)}

The effect of semaglutide on body weight was reported as a secondary endpoint in the SUSTAIN programme and both doses of semaglutide demonstrated significant reductions in weight after 30-56 weeks. Semaglutide $0.5 \mathrm{mg} \mathrm{QW}$ was associated with 3.5-4.6 Kg weight loss in the trials and this is equivalent to a $3.7-4.8 \%$ reduction of baseline body mass. At a dose of $1.0 \mathrm{mg} \mathrm{QW}$, semaglutide achieved weight reductions of 4.5-6.5 $\mathrm{Kg}$ (4.7-7.0\% baseline body mass). The observed reductions in weight with semaglutide $0.5 \mathrm{mg} \mathrm{QW}$ and $1.0 \mathrm{mg}$ QW were significantly better than those seen with comparators, placebo, sitagliptin $100 \mathrm{mg}$ OD and insulin glargine (REF 19)._Semaglutide $1.0 \mathrm{mg} \mathrm{QW}$ also resulted in greater weight reductions than the other once weekly GLP-1RAs, exenatide ER and dulaglutide (REF 20 , 23). Significant weight loss was also seen when semaglutide was added to basal insulin therapy, at least partly due to the reduction in insulin dose in those subjects receiving semaglutide (REF 22).

\section{Secondary endpoints - Blood pressure and pulse rate}


In the SUSTAIN phase 3 clinical trial programme, treatment with semaglutide $0.5 \mathrm{mg}$ QW was associated with a fall in systolic blood pressure (SBP) of 2.4-5.1 mm Hg whilst the 1.0 $\mathrm{mg}$ QW dose led to a fall of $2.7-6.3 \mathrm{~mm} \mathrm{Hg}$. This effect was significantly greater than the comparators except for placebo in SUSTAIN 1 (REF 18) and dulaglutide in SUSTAIN 7 (REF 23). Diastolic blood pressure was also lowered although to a lesser extent, and generally not significantly different from comparators (apart from SUSTAIN 7 where semaglutide achieved a larger reduction than dulaglutide (REF 23).

Across the SUSTAIN programme, semaglutide was associated with an increase in pulse rate of 1-4 beats per minute, a finding which has been consistently observed with all GLP-1RAs (REF 24). In the head-to-head comparisons (SUSTAIN 3 and 7), semaglutide $1.0 \mathrm{mg}$ QW had a greater increase in heart rate than dulaglutide $1.5 \mathrm{mg}$ QW (4.0 versus 2.4 beats per $\min )($ REF 23).

\section{Secondary endpoints - Other cardiovascular risk factors}

The impact of semaglutide on plasma lipids is marginal but where changes are seen, these are generally in a positive direction (i.e. favouring CV protection). In SUSTAIN 1 and 4, a significant reduction in total cholesterol and low-density lipoprotein (LDL) was reported (REF 18, 21), whilst there were reductions in free fatty acids in SUSTAIN 1 and 3 (REF 18,20), and falls in triglycerides in SUSTAIN 3 and 4 (REF 19,21). Finally, high-density lipoprotein (HDL) significantly increased and very low-density lipoprotein (VLDL) fell in subjects randomised to semaglutide in SUSTAIN 2 (REF 19). C-reactive protein (a biomarker for CV risk)(REF 25), was measured in SUSTAIN 2 and 4 and was found to be significantly reduced by semaglutide (both $0.5 \mathrm{mg}$ and $1.0 \mathrm{mg} \mathrm{QW})(\mathrm{REF} 19,21)$.

\section{SUSTAIN 6 - Cardiovascular outcomes trial}

In 2008 the United States FDA mandated that all new glucose lowering therapies should be subjected to a cardiovascular outcomes trial (CVOT) to demonstrate CV safety (REF 26). Semaglutide was assessed in the SUSTAIN 6 trial (REF 27), which was performed before drug approval and was, therefore, smaller and shorter than some other CVOTs for GLP-RAs, such as LEADER (liraglutide) (REF 28) and EXSCEL (exenatide ER)(REF 29). SUSTAIN 6 was a randomised, double-blind, placebo-controlled, multicentre study conducted between 20132016. Subjects were allocated to semaglutide, $0.5 \mathrm{mg}$ and $1.0 \mathrm{mg} \mathrm{QW}$ or volume-matched placebo, in addition to CV 'standard of care'. Standard of care promoted optimal management of blood pressure, lipids and use of anti-platelet therapies, as well as aiming for equivalent glucose lowering in each of the trial arms (so-called 'glycaemic equipoise' (REF 30)). SUSTAIN 6 enrolled 3,297 people with T2DM at high CV risk and was both event and time-driven, patients having a minimum of two years exposure to investigational medical product (IMP). Recruitment criteria were similar to those of the LEADER trial with two cohorts: 1 ) age $\geq 50$ years and established CV disease (cerebrovascular disease, coronary heart disease, peripheral vascular disease, chronic kidney disease (stage 3 or higher), or chronic heart failure of New York Heart Association classes II-III); 2) age $>60$ years and one or more CV risk factors (for example, microalbuminuria, hypertension). The primary outcome was a composite of major cardiovascular events (MACE); CV death, non-fatal 
myocardial infarction or non-fatal stroke. Despite the attempt to achieve glycaemic equipoise (which led to more glucose lowering therapies being initiated in the placebo arms), semaglutide $0.5 \mathrm{mg} \mathrm{QW}$ and $1.0 \mathrm{mg} \mathrm{QW}$ significantly lowered $\mathrm{HbA} 1 \mathrm{c}$ by 7.7 $\mathrm{mmol} / \mathrm{mol}$ and $11.1 \mathrm{mmol} / \mathrm{mol}$ respectively. Weight was also significantly reduced by 2.87 $4.35 \mathrm{Kg}$ by semaglutide.

The primary endpoint occurred in fewer people receiving semaglutide than placebo $(6.6 \% \mathrm{vs}$ $8.9 \%)$ giving a hazard ratio (HR) of 0.74 (95\% confidence intervals $0.58-0.95$ ) which demonstrated statistically significant non-inferiority for the CV safety of semaglutide. A test for superiority of CV safety for semaglutide (which was not pre-specified) was also significant and was driven by a reduction in nonfatal stroke (seen in $1.6 \%$ of the patients in the semaglutide group versus $2.7 \%$ for placebo). Hospitalisation rates for heart failure were similar in the two groups. As a result of the SUSTAIN 6 trial, semaglutide is one of the preferred GLP-1RAs for second-line use in people with T2DM and very high CV risk (REF 31,32).

\section{Safety}

In the SUSTAIN phase 3 trial programme, the rates of discontinuation of IMP due to adverse events (AEs) were low, amounting to $5-13 \%$ in SUSTAIN 1-5 and 7. There was a higher discontinuation rate in SUSTAIN 6 (around 20\%) but this trial was of a much longer duration. Discontinuation rates was higher for semaglutide than placebo, sitagliptin and insulin glargine (SUSTAIN 2, 4 and 5), but were comparable in the head-to-head GLP-1RA studies (SUSTAIN 3 and 7). The predominant AEs of semaglutide were GI and are consistent with the known side-effects of this class of glucose lowering therapy (REF 18-23, 28,33).Nausea was reported by $11-24 \%$ of people receiving semaglutide, diarrhoea was reported by $5-19 \%$ and vomiting by $4-14 \%$.

Frequency of hypoglycaemia (severe, requiring third-party assistance, or confirmed blood glucose $<3.1 \mathrm{mmol} / \mathrm{L}$ ) was very low in SUSTAIN trials (1-2\%) unless semaglutide was coadministered with insulin or sulphonylureas (REF 18,19,23). In trials including these agents with their known potential for hypoglycaemia, rates were between 4-10.7\% (REF 20-22).

In the SUSTAIN 6 CVOT there was an increase in the rate of diabetic retinopathy (DR) events observed with semaglutide versus placebo (3.0\% versus $1.8 \%, \mathrm{HR} 1.76)$ and this was statistically significant (REF 27). The reason for this finding has not been fully explained, but interestingly there was no signal for increased DR in SUSTAIN 1-5 and 7, trials which excluded participants with active DR and had an upper threshold of $\mathrm{HbA1c}$ for inclusion. This has led to the suggestion that worsening of DR is caused by a rapid reduction in glucose levels in subjects with very poor glycaemic control and existing retinopathy (REF 34,35). It is perhaps of note that a non-significant increase in DR events was also seen in the CVOTs for liraglutide and dulaglutide (REF 28,36) consistent with an impact of potent glucoselowering. There was no evidence of deterioration in renal events in SUSTAIN 6, another long-term diabetes complication which is regarded as being microvascular, indeed the onset of new or worsening albuminuria was lower in semaglutide treated patients (REF 27). 
As has been extensively documented for liraglutide, serum amylase and lipase levels increased during exposure to semaglutide, however, the incidence of pancreatitis was low in the SUSTAIN programme $(<1 \%)$ and similar to comparator treatments.

\section{Post-approval studies (table)}

Since semaglutide by injection (Ozempic ${ }^{\circledR}$ ) was approved in the United States in 2017, the SUSTAIN trial programme has completed additional clinical trials, with three being published in 2019 (REF 37-39). These studies have confirmed the previously reported efficacy of semaglutide, in terms of glucose and weight lowering, without raising any new safety concerns. More importantly, they have provided additional data regarding the performance of semaglutide compared with other commonly used glucose-lowering therapies and in combination with a sodium-glucose co-transporter-2 (SGLT-2) inhibitor.

\section{SUSTAIN 8}

This was a double-blind, parallel-group, RCT where people with sub-optimally controlled T2DM were assigned to semaglutide $1.0 \mathrm{mg}$ QW or oral canagliflozin (an SGLT-2 inhibitor) $300 \mathrm{mg}$ OD(REF 37). Participants randomised to semaglutide had a significantly greater lowering of both $\mathrm{HbA} 1 \mathrm{c}$ and body weight compared with those receiving canagliflozin ( $\mathrm{HbA} 1 \mathrm{c}$ estimated treatment difference [ETD]-5.34 mmol/mol and body weight ETD -1.06 $\mathrm{Kg}$ ). GI disorders, mainly nausea, were the most frequently reported AE with semaglutide, occurring in $47 \%$ of subjects; premature treatment discontinuation because of AEs occurred in $10 \%$ of semaglutide patients compared with $5 \%$ on canagliflozin.

\section{SUSTAIN 9}

This trial investigated the safety and efficacy of semaglutide $1.0 \mathrm{mg}$ QW added to an SGLT-2 inhibitor compared with placebo (REF 38). In addition to IMP and SGLT-2 inhibitor, $71.5 \%$ of trial participants were taking metformin and $12.9 \%$ a sulphonylurea. At EOT (30 weeks) semaglutide produced significantly larger reductions in mean $\mathrm{HbA}_{1 \mathrm{c}}$ (ETD $-15.55 \mathrm{mmol} / \mathrm{mol}$ ) and body weight $(-3.81 \mathrm{Kg})$. GI AEs were reported in $37.3 \%$ people in the semaglutide group and $13.2 \%$ for placebo. Hypoglycaemia (severe or blood glucose-confirmed) was reported in $2.7 \%$ patients on semaglutide.

\section{SUSTAIN 10}

This study compared the safety and efficacy of semaglutide $1.0 \mathrm{mg}$ QW with the most frequently prescribed dose of liraglutide in European clinical practice (1.2 mg OD)(REF 39). The trial was open label and included people with sub-optimally controlled T2DM on 1-3 oral glucose lowering therapies. Mean HbA1c at EOT (30 weeks) fell from $66.1 \mathrm{mmol} / \mathrm{mol}$ to $47.5 \mathrm{mmol} / \mathrm{mol}$ with semaglutide and to $55.2 \mathrm{mmol} / \mathrm{mol}$ with liraglutide (ETD 7.7 $\mathrm{mmol} / \mathrm{mol}$ ) whilst the reduction in weight (ETD $-3.83 \mathrm{Kg}$ ) also significantly favoured semaglutide. Both therapies had similar safety profiles, but with more frequent GI AEs with semaglutide (43.9\% vs. $38.3 \%)$.

\section{Conclusion}

GLP-1RA therapies have been available for the treatment of people with T2DM since 2006 and are being increasingly used by clinicians due to their efficacy in glucose lowering and 
weight reduction. There is also evidence for CV protection in GLP-1RAs which are analogues of human GLP-1.

Semaglutide is the most recently approved GLP-1RA and has $94 \%$ homology with the human GLP-1 molecule. It is one of three currently available GLP-1RAs which can be administered once weekly. It has been assessed in an extensive phase 3 clinical trial programme, spanning the continuum of people with T2DM from treatment naïve to those receiving insulin. It demonstrates high efficacy in reducing hyperglycaemia, as assessed by $\mathrm{HbA1C}$, and has additional benefits of weight reduction and blood pressure lowering. In terms of $\mathrm{HbA1C}$ and weight, it is significantly more potent than a DPP4 inhibitor (sitagliptin), a SGLT-2 inhibitor (canaglifozin) and basal insulin (glargine). It has also been assessed in head-to-head comparisons with three GLP-1RAs (including both the other weekly agents, exenatide ER and dulaglutide) and been found to be significantly more efficacious in terms of both $\mathrm{HbA1c}$ and weight reduction.

The CVOT for semaglutide (SUSTAIN 6) demonstrated the CV safety of this medicine and a significant $26 \%$ reduction in the primary end-point of 3-point MACE (albeit in a nonprespecified analysis).

The AE profile of semaglutide is as expected for a GLP-1RA therapy, with increased GI upset including nausea, vomiting and diarrhoea. These symptoms tend to diminish with time and are reduced by slow up-titration of drug. However, on balance there does appear to be more Gl upset with semaglutide compared with other GLP-1RAs and this may affect adherence in clinical practice. The unexpected finding of an increase in retinopathy events in the SUSTAIN 6 trial may be explained by potent glucose lowering in patients with poor glycaemic control and pre-existing DR; caution should be urged when initiating semaglutide (and probably all GLP-1RAs) in these cases.

\section{Keywords:}

CVOT, Glucagon-like peptide-1, GLP-1RA, Semaglutide, SUSTAIN, Type 2 diabetes, T2DM

\section{Article highlights:}

- Semaglutide is the most recently approved once weekly injectable GLP-1 receptor agonist for the treatment of type 2 diabetes

- Semaglutide is more efficacious in reducing $\mathrm{HbA1c}$ and weight than other classes of glucose lowering therapies

- Head-to-head comparisons with other GLP-1RAs, including exenatide ER and dulaglutide, show superiority of semaglutide for both $\mathrm{HbA} 1 \mathrm{c}$ and weight reduction

- The cardiovascular outcome trial for semaglutide showed both safety and a reduction in major cardiovascular events

- Gastrointestinal side effects are commonly seen with semaglutide and may be more frequent than with other GLP-1RA therapies

- Caution is needed when initiating semaglutide in patient with pre-existing diabetic retinopathy and poor glycaemic control 


\section{Expert opinion:}

Glucagon-like peptide-1 receptor agonists (GLP-1Ras) are now a well-established class of injectable peptides for the management of people with type 2 diabetes (T2DM). They have a high level of efficacy in reducing hyperglycaemia and the secondary benefits of weight reduction and blood pressure lowering. Indeed, a high dose formulation of liraglutide is now licenced for the management of obesity. GLP-1RAs, which are based on the human GLP-1 molecule (rather than exendin-4), have been shown to reduce major adverse cardiovascular events in people with T2DM and, as a result of these findings, are now positioned earlier in the treatment algorithm of people with T2DM and high cardiovascular risk. The side effects of GLP-1RAs are typically gastrointestinal (GI) with nausea, vomiting and diarrhoea, especially at the time of initiation. These can be limited by slow up-titration of drug and tend to diminish over time. Early concerns regarding rises in amylase and lipase and the potential for pancreatitis (and even pancreatic cancer) have not been realised (REF 40), nor has there been any clinical evidence of an increase in medullary thyroid cancer (which was seen in rodents during pre-clinical studies of liraglutide) (REF 41).

Semaglutide is the most recently approved GLP-1RA and has $94 \%$ homology with human GLP-1. It is very similar to liraglutide, having one additional amino acid difference and a longer di-acid fatty chain, allowing for tight binding to albumin and the protracted duration of action. Semaglutide thus becomes forth once weekly GLP-1RA to be approved and is one of three currently marketed (albiglutide having been withdrawn for commercial reasons in 2018 (REF 42). The use of once weekly GLP-1RAs is preferred by patients since it reduces the burden of injections and this is reflected in the increasing market share for these agents. Semaglutide has been trialled against both of the other weekly GLP-1RAs (exenatide ER and dulaglutide) and has been shown to be superior in both $\mathrm{HbA} 1 \mathrm{c}$ and weight reduction. It also has a supportive cardiovascular outcomes trial (CVOT), which showed a significant 26\% reduction in the primary composite end-point of cardiovascular death, nonfatal myocardial infarction and nonfatal stroke (albeit in a non-prespecified statistical analysis); the CVOT for dulaglutide (REWIND) was also positive, whilst that for exenatide ER narrowly missed significance. Based on these data, it seems likely that semaglutide will become the once weekly GLP-1RA of choice going forwards.

Are there any downsides? There is a suggestion that the Gl side-effects with semaglutide may be more frequent and possibly more severe than with the other GLP-RAs and this may impact on adherence. Also, the increased diabetic retinopathy events seen with semaglutide in SUSTAIN 6 will concern some prescribers. It is of note that no signal for retinopathy events was seen in the SUSTAIN 1-5 and 7 trials of semaglutide, which all had an upper limit of HbA1c for inclusion and excluded patients with active retinopathy. In our view, it is likely that the worsening of diabetic retinopathy reported in SUSTAIN 6 was related to rapid reduction of hyperglycaemia in subjects with poor glucose control and significant existing eye disease. This has been seen previously with insulin treatment and following bariatric surgery. It is also consistent with the non-significant increase in DR events seen in the CVOTs for both liraglutide and dulaglutide, which are also potent glucose-lowering medications.

Other aspects will also impact on clinical decision-making, for example the injection preparation (problematic for exenatide ER, which requires vigorous shaking prior to 
injection) as well as the design of administration device, which may favour dulaglutide (REF 43). And there is also the issue of cost, which will vary in different countries. Where the prices of the long-acting GLP-1RAs are equivalent, then health technology analyses are largely driven by HbA1c and weight and reflect differences in efficacy (REF 44).

It is possible, however, that the biggest threat to the future dominance of semaglutide in the GLP-1RA market comes from itself. Oral semaglutide has been developed as a tablet, coformulated with an absorption enhancer known as SNAC (sodium N-(8-[2-hydroxylbenzoyl] amino) caprylate)(REF 45). SNAC facilitates the passage of semaglutide across the gastric mucosa via a transcellular mechanism, as well as providing a localised increase in $\mathrm{pH}$ which protects the semaglutide from degradation (REF 46). The pharmacokinetics of oral semaglutide allow for once daily dosing and the results from the phase 3 clinical trial programme (named PIONEER) are extremely impressive. Oral semaglutide is significantly more effective in reducing $\mathrm{HbA1c}$ than placebo (REF 47), sitagliptin (REF 48), empagliflozin (REF 49) and injected liraglutide (REF 50), as well as having a substantial impact of weight reduction. A CVOT of oral semaglutide (PIONEER 6)(REF 51,52) has shown cardiovascular safety and a superiority trial for CV events (SOUL) is on-going (REF 53). There is a possibility that the oral version of semaglutide, approved for use in the United States in 2019 and the EU in 2020, might usurp the prominence of the injectable version, before this has become fully established....

\section{References}

REF 1. Porte D, Kahn SE. Beta-cell dysfunction and failure in type 2 diabetes: potential mechanisms. Diabetes. 2001;50(Suppl 1):S160-S163. doi: 10.2337/diabetes.50.2007.s160.

REF 2. Saeedi P, Petersohn I, Salpea P, Malanda B, Karuranga S, Unwin N, Colagiuri S, Guariguata L, Motala AA, Ogurtsova K, Shaw JE, Bright D, Williams R; IDF Diabetes Atlas Committee. Global and regional diabetes prevalence estimates for 2019 and projections for 2030 and 2045: Results from the International Diabetes Federation Diabetes Atlas, 9th edition. Diabetes Res Clin Pract. 2019 Nov;157:107843. doi: 10.1016/j.diabres.2019.107843.

REF 3. https://www.ema.europa.eu/en/medicines/human/EPAR/byetta

REF 4. https://www.ema.europa.eu/en/medicines/human/EPAR/bydureon

REF 5. https://www.ema.europa.eu/en/medicines/human/EPAR/trulicity

REF 6. https://www.ema.europa.eu/en/medicines/human/EPAR/ozempic

REF 7. Dhillon S. Semaglutide: first global approval. Drugs. 2018;78(2):275-284. doi: 10.1007/s40265-018-0871-0.

REF 8. Jensen L, Helleberg $H$, Roffel A, et al. Absorption, metabolism and excretion of the GLP-1 analogue semaglutide in humans and nonclinical species. Eur J Pharm Sci Off J Eur Fed Pharm Sci. 2017;104:31-41. doi: 10.1016/j.ejps.2017.03.020. 
REF 9. Marbury TC, Flint A, Jacobsen JB, et al. Pharmacokinetics and tolerability of a single dose of semaglutide, a human glucagon-like peptide-1 analog, in subjects with and without renal impairment. Clin Pharmacokinet. 2017;56(11):1381-1390. doi: 10.1007/s40262-0170528-2

REF 10. Blundell J, Finlayson $G$, Axelsen $M$, et al. Effects of once-weekly semaglutide on appetite, energy intake, control of eating, food preference and body weight in subjects with obesity. Diabetes Obes Metab. 2017;19(9):1242-51.

REF 11. Kapitza C, Nosek L, Jensen L, Hartvig H, Jensen CB, Flint A. Semaglutide, a onceweekly human GLP-1 analog, does not reduce the bioavailability of the combined oral contraceptive, ethinylestradiol/levonorgestrel. J Clin Pharmacol. 2015;55(5):497-504.

REF 12. Lau J, Bloch $P$, Schaffer $L$, et al. Discovery of the once-weekly glucagon-like peptide-1 (GLP-1) analogue semaglutide. J Med Chem. 2015;58(18):7370-80.

REF 13. Victoza (liraglutide) [prescribing information]. Bagsvaerd: Novo Nordisk. 2017. http://www.novo-pi.com/victoza.pdf.

REF 14. Trulicity (dulaglutide) [prescribing information]. Indianapolis: Eli Lily and Company. 2017. http://pi.lilly.com/us/trulicity-uspi. pdf.

REF 15. Gedulin BR, Smith PA, Jodka CM, et al. Pharmacokinetics and pharmacodynamics of exenatide following alternate routes of administration. Int J Pharm. 2008;356(1-2):231-8.

REF 16. Nauck MA, Petrie JR, Sesti G, Mannucci E, Courrèges JP, Lindegaard ML, Jensen CB, Atkin SL \& Study 1821 Investigators. A Phase 2, Randomized, Dose-Finding Study of the Novel Once-Weekly Human GLP-1 Analog, Semaglutide, Compared With Placebo and OpenLabel Liraglutide in Patients With Type 2 Diabetes. Diabetes Care. 2016 Feb;39(2):231-41. doi: 10.2337/dc15-0165.

REF 17. Goldenberg RM, Steen O. Semaglutide: Review and Place in Therapy for Adults With Type 2 Diabetes. Can J Diabetes. 2019 Mar;43(2):136-145. doi: 10.1016/j.jcjd.2018.05.008.

REF 18. Sorli C, Harashima S-I, Tsoukas GM, et al. Efficacy and safety of once-weekly semaglutide monotherapy versus placebo in patients with type 2 diabetes (SUSTAIN 1): a double-blind, randomised, placebo-controlled, parallel-group, multinational, multicentre phase 3a trial. Lancet Diabetes Endocrinol. 2017;5(4):251-260. doi: 10.1016/S22138587(17)30013-X.

REF 19. Ahrén B, Masmiquel L, Kumar $H$, et al. Efficacy and safety of once-weekly semaglutide versus once-daily sitagliptin as an add-on to metformin, thiazolidinediones, or both, in patients with type 2 diabetes (SUSTAIN 2): a 56-week, double-blind, phase 3a, randomised trial. Lancet Diabetes Endocrinol. 2017;5(5):341-354. doi: 10.1016/S22138587(17)30092-X. 
*REF 20. Ahmann AJ, Capehorn M, Charpentier G, et al. Efficacy and safety of once-weekly semaglutide versus exenatide ER in subjects with type 2 diabetes (SUSTAIN 3): a 56-week, open-label, randomized clinical trial. Diabetes Care. 2018;41(2):258-266. doi: 10.2337/dc170417.

The first head-to-head efficacy trial of semaglutide against another once-weekly GLP-1RA (exenatide ER).

REF 21. Aroda VR, Bain SC, Cariou B, et al. Efficacy and safety of once-weekly semaglutide versus once-daily insulin glargine as add-on to metformin (with or without sulfonylureas) in insulin-naive patients with type 2 diabetes (SUSTAIN 4): a randomised, open-label, parallelgroup, multicentre, multinational, phase $3 a$ trial. Lancet Diabetes Endocrinol. 2017;5(5):355-366. doi: 10.1016/S2213-8587(17)30085-2.

REF 22. Rodbard HW, Lingvay I, Reed J, et al. Semaglutide added to basal insulin in type 2 diabetes (SUSTAIN 5): a randomized, controlled trial. J Clin Endocrinol Metab. 2018;103(6):2291-2301. doi: 10.1210/jc.2018-00070.

REF 23. Pratley RE, Aroda VR, Lingvay I, et al. Semaglutide versus dulaglutide once weekly in patients with type 2 diabetes (SUSTAIN 7): a randomised, open-label, phase $3 \mathrm{~b}$ trial. Lancet Diabetes Endocrinol. 2018;6(4):275-286. doi: 10.1016/S2213-8587(18)30024-X.

The second head-to-head efficacy trial of semaglutide against another once-weekly GLP-1RA (dulaglutide)

REF 24. Smits MM, Tonneijck L, Muskiet MH, Hoekstra T, Kramer MH, Diamant M, van Raalte DH. Heart rate acceleration with GLP-1 receptor agonists in type 2 diabetes patients: an acute and 12-week randomised, double-blind, placebo-controlled trial. Eur J Endocrinol. 2017 Jan;176(1):77-86.

REF 25. Koenig W. High-sensitivity C-reactive protein and atherosclerotic disease: from improved risk prediction to risk-guided therapy. Int J Cardiol. 2013;168(6):5126-5134. doi: 10.1016/j.ijcard.2013.07.113.

REF 26. Guidance for industry diabetes mellitus-evaluating cardiovascular risk in new antidiabetic therapies to treat type 2 diabetes. US Department of Health and Human Services, Washington, DC: FDA/Center for Drug Evaluation, 2008. Available at: http://www.fda.gov/downloads/drugs/guidancecomplianceregulatoryinformation/guidance s/ucm071627.pdf

**REF 27. Marso SP, Bain SC, Consoli A, et al. Semaglutide and cardiovascular outcomes in patients with type 2 diabetes. N Engl J Med. 2016;375(19):1834-1844. doi: 10.1056/NEJMoa1607141

The SUSTAIN 6 CVOT for subcutaneous semaglutide showing a 26\% reduction in MACE versus placebo, the second trial for a GLP-1RA to show this benefit (following the LEADER trial for liraglutide). 
REF 28. Marso SP, Daniels GH, Brown-Frandsen K, et al. Liraglutide and cardiovascular outcomes in type 2 diabetes. N Engl J Med. 2016;375(4):311-322. doi: 10.1056/NEJMoa1603827.[

REF 29. Holman RR, Bethel MA, Mentz RJ, Thompson VP, Lokhnygina Y, Buse JB, Chan JC, Choi J, Gustavson SM, Iqbal N, Maggioni AP, Marso SP, Öhman P, Pagidipati NJ, Poulter N, Ramachandran A, Zinman B, Hernandez AF; EXSCEL Study Group. Effects of Once-Weekly Exenatide on Cardiovascular Outcomes in Type 2 Diabetes. N Engl J Med. 2017 Sep 28;377(13):1228-1239. doi: 10.1056/NEJMoa1612917.

REF 30. Kalra S, John M, Unnikrishnan AG. Glycemic equipoise. Indian J Endocrinol Metab. 2017 Jan-Feb;21(1):18-20. doi: 10.4103/2230-8210.194361

**REF 31. Davies MJ, D'Alessio DA, Fradkin J, et al. Management of hyperglycaemia in type 2 diabetes, 2018. A consensus report by the American Diabetes Association (ADA) and the European Association for the Study of Diabetes (EASD) Diabetologia. 2018 doi: 10.1007/s00125-018-4729-5.

A consensus statement for the management of type 2 diabetes which takes into account modern CVOTs

REF 32. Cosentino F, Grant PJ, Aboyans V, Bailey CJ, Ceriello A, Delgado V, Federici M, Filippatos G, Grobbee DE, Hansen TB, Huikuri HV, Johansson I, Jüni P, Lettino M, Marx N, Mellbin LG, Östgren CJ, Rocca B, Roffi M, Sattar N, Seferović PM, Sousa-Uva M, Valensi P, Wheeler DC; ESC Scientific Document Group. 2019 ESC Guidelines on diabetes, pre-diabetes, and cardiovascular diseases developed in collaboration with the EASD. Eur Heart J. 2019 Aug 31. pii: ehz486. doi: 10.1093/eurheartj/ehz486.

REF 33. Trujillo JM, Nuffer W, Ellis SL. GLP-1 receptor agonists: a review of head-to-head clinical studies. Ther Adv Endocrinol Metab. 2015;6(1):19-28. doi: $10.1177 / 2042018814559725$

REF 34. Vilsbøll T, Bain SC, Leiter LA, Lingvay I, Matthews D, Simó R, Helmark IC, Wijayasinghe $N$, Larsen $M$. Semaglutide, reduction in glycated haemoglobin and the risk of diabetic retinopathy. Diabetes Obes Metab. 2018 Apr;20(4):889-897. doi: 10.1111/dom.13172.

REF 35. Bain SC, Klufas MA, Ho A, Matthews DR. Worsening of diabetic retinopathy with rapid improvement in systemic glucose control: A review. Diabetes Obes Metab. 2019 Mar;21(3):454-466. doi: 10.1111/dom.13538.

REF 36. Gerstein HC, Colhoun HM, Dagenais GR, Diaz R, Lakshmanan M, Pais P, Probstfield J, Riesmeyer JS, Riddle MC, Rydén L, Xavier D, Atisso CM, Dyal L, Hall S, Rao-Melacini P, Wong G, Avezum A, Basile J, Chung N, Conget I, Cushman WC, Franek E, Hancu N, Hanefeld M, Holt S, Jansky P, Keltai M, Lanas F, Leiter LA, Lopez-Jaramillo P, Cardona Munoz EG, Pirags V, Pogosova N, Raubenheimer PJ, Shaw JE, Sheu WH, Temelkova-Kurktschiev T; REWIND Investigators. Dulaglutide and cardiovascular outcomes in type 2 diabetes (REWIND): a 
double-blind, randomised placebo-controlled trial. Lancet. 2019 Jul 13;394(10193):121-130. doi: 10.1016/S0140-6736(19)31149-3.

REF 37. Lingvay I, Catarig AM, Frias JP, Kumar H, Lausvig NL, le Roux CW, Thielke D, Viljoen A, McCrimmon RJ. Efficacy and safety of once-weekly semaglutide versus daily canagliflozin as add-on to metformin in patients with type 2 diabetes (SUSTAIN 8): a double-blind, phase 3b, randomised controlled trial. Lancet Diabetes Endocrinol. 2019 Nov;7(11):834-844. doi: 10.1016/S2213-8587(19)30311-0.

REF 38. Zinman B, Bhosekar V, Busch R, et al. Semaglutide once weekly as add-on to SGLT-2 inhibitor therapy in type 2 diabetes (SUSTAIN 9): a randomised, placebo-controlled trial. Lancet Diabetes Endocrinol. 2019;7(5):356-367. doi: 10.1016/S2213-8587(19)30066-X.

REF 39. Capehorn MS, Catarig AM, Furberg JK, Janez A, Price HC4, Tadayon S2, Vergès B5, Marre M6.Efficacy and safety of once-weekly semaglutide $1.0 \mathrm{mg}$ vs once-daily liraglutide $1.2 \mathrm{mg}$ as add-on to 1-3 oral antidiabetic drugs in subjects with type 2 diabetes (SUSTAIN 10). Diabetes Metab. 2019 Sep 17:101117. doi: 10.1016/j.diabet.2019.101117.

REF 40. Bjerre Knudsen L, Madsen LW, Andersen S, et al. Glucagon-like Peptide-1 receptor agonists activate rodent thyroid C-cells causing calcitonin release and C-cell proliferation. Endocrinology. 2010;151(4):1473-1486. doi: 10.1210/en.2009-1272

REF 41. Monami M, Nreu B, Scatena A, Cresci B, Andreozzi F, Sesti G, Mannucci E. Safety issues with glucagon-like peptide-1 receptor agonists (pancreatitis, pancreatic cancer and cholelithiasis): Data from randomized controlled trials. Diabetes Obes Metab. 2017 Sep;19(9):1233-1241. doi: 10.1111/dom.12926.

REF 42.

https://assets.publishing.service.gov.uk/media/59f081c6e5274a18bc07411b/Eperzan_Lette r_-_090917.pdf

REF 43. Boye KS, Matza LS, Stewart KD, Jordan J, Biricolti G, Del Santo S, Perez-Nieves M, Federici MO, Gentilella R, Losi S, Norrbacka K. Patient preferences and health state utilities associated with dulaglutide and semaglutide injection devices among patients with type 2 diabetes in Italy. J Med Econ. 2019 Aug;22(8):806-813. doi: 10.1080/13696998.2019.1609482.

REF 44. Viljoen A, Hoxer CS, Johansen P, Malkin S, Hunt B, Bain SC. Evaluation of the longterm cost-effectiveness of once-weekly semaglutide versus dulaglutide for treatment of type 2 diabetes mellitus in the UK. Diabetes Obes Metab. 2019 Mar;21(3):611-621. doi: 10.1111/dom.13564.

**REF 45. Davies M, Pieber TR, Hartoft-Nielsen ML,Hansen OKH, Jabbour S, Rosenstock J. Effect of oral semaglutide compared with placebo and subcutaneous semaglutide on glycemic control in patients with type 2 diabetes: a randomized clinical trial. JAMA 2017;318:1460-1470 
The original clinical publication on oral semaglutide use, which demonstrates proof of concept for the oral preparation and also includes an injectable semaglutide arm.

REF 46. Buckley ST, Bækdal TA, Vegge A, et al. Transcellular stomach absorption of a derivatized glucagon-like peptide-1 receptor agonist. Sci Transl Med 2018;10:eaar7047

REF 47. Aroda VR, Rosenstock J, Terauchi Y, et al. PIONEER 1: randomized clinical trial of the efficacy and safety of oral semaglutide monotherapy in comparison with placebo in patients with type 2 diabetes. Diabetes Care. 2019;42(9):1724-1732. doi: 10.2337/dc19-0749.

REF 48. Rosenstock J, Allison D, Birkenfeld AL, et al. Effect of additional oral semaglutide vs sitagliptin on glycated hemoglobin in adults with type 2 diabetes uncontrolled with metformin alone or with sulfonylurea: the PIONEER 3 randomized clinical trial. JAMA. 2019;321(15):1466-1480. doi: 10.1001/jama.2019.2942.

REF 49. Rodbard HW, Rosenstock J, Canani LH, et al. Oral semaglutide versus empagliflozin in patients with type 2 diabetes uncontrolled on metformin: the PIONEER 2 trial. Diabetes Care. 2019 doi: 10.2337/dc19-0883.

REF 50. Pratley R, Amod A, Hoff ST, et al. Oral semaglutide versus subcutaneous liraglutide and placebo in type 2 diabetes (PIONEER 4): a randomised, double-blind, phase 3a trial. Lancet Lond Engl. 2019;394(10192):39-50. doi: 10.1016/S0140-6736(19)31271-1.

REF 51. Bain SC, Mosenzon O, Arechavaleta R, et al. Cardiovascular safety of oral semaglutide in patients with type 2 diabetes: rationale, design and patient baseline characteristics for the PIONEER 6 trial. Diabetes Obes Metab. 2019;21(3):499-508. doi: 10.1111/dom.13553.

**REF 52. Husain M, Birkenfeld AL, Donsmark M, et al. Oral semaglutide and cardiovascular outcomes in patients with type 2 diabetes. N Engl J Med. 2019;381(9):841-851. doi: 10.1056/NEJMoa1901118.

The CVOT for oral semaglutide, which adds additional data for the CV safety of the molecule to that obtained for injectable semaglutide in the SUSTAIN 6 CVOT.

REF 53. https://clinicaltrials.gov/ct2/show/NCT03914326 